Archives

\title{
La compagnie de jésus et le souvenir du vœu de montmartre (1534)
}

État d'une recherche

Pierre-Antoine Fabre

\section{(2) OpenEdition \\ Journals}

Édition électronique

URL : http://journals.openedition.org/ccrh/2032

DOI : $10.4000 /$ ccrh.2032

ISSN : $1760-7906$

Éditeur

Centre de recherches historiques - EHESS

Édition imprimée

Date de publication : 20 avril 2000

ISSN : 0990-9141

Référence électronique

Pierre-Antoine Fabre, «La compagnie de jésus et le souvenir du vœu de montmartre (1534)», Les

Cahiers du Centre de Recherches Historiques [En ligne], 24 | 2000, mis en ligne le 17 janvier 2009,

consulté le 10 décembre 2020. URL : http://journals.openedition.org/ccrh/2032 ; DOI : https://doi.org/ $10.4000 /$ ccrh.2032

Ce document a été généré automatiquement le 10 décembre 2020.

Article L.111-1 du Code de la propriété intellectuelle. 


\title{
La compagnie de jésus et le souvenir du vœu de montmartre (1534)
}

\author{
État d'une recherche
}

Pierre-Antoine Fabre

1 On pose dans les pages qui suivent quelques jalons d'une recherche en cours sur la fonction d'un rituel polymorphe, dit "vœu de Montmartre", dans la tradition historiographique de l'ancienne Compagnie de Jésus, et sur ses implications dans la réélaboration jésuite de la théorie et de la pratique du vœu de religion au cours de la seconde moitié du $\mathrm{XvI}^{\mathrm{e}}$ siècle ${ }^{1}$. Pourquoi un rituel? Accompli une fois (autrefois et une seule fois), le vœu de Montmartre est cependant rappelé et commémoré - et, comme on le verra, ne se donne à connaître que dans la mesure et dans la distance de ce souvenir, de ce retardement : il nous semble porteur par essence d'une capacité répétitive, c'est-à-dire d'une efficacité attachée à sa reproduction et solidaire de celle-ci et, à partir de là, relève donc du rite. Pourquoi un rituel polymorphe ? L'événement unique du vœu de Montmartre est marqué, en revanche, et c'est cet aspect peu envisagé qui nous retiendra le plus, par une pluralité de significations possibles, ouvertes par une pluralité de points de vue sur l'événement, qui donnent à celui-ci, selon chaque témoignage, une forme particulière. Le vœu de Montmartre, qui est un fait de mémoire, est la composition hétérogène de plusieurs donations de sens toutes figurées par un modèle spécifique. Le rituel de ce vœu est donc la célébration d'une mémoire plurielle, doublement déterminée par une multiplicité de significations et une multiplicité de modèles rituels.

2 Trois problèmes seront abordés : le vœu de Montmartre comme fait historique (comment le définir, ou comment le construire et l'établir ?), la polyphonie du vœu de Montmartre, l'inscription historique du fait, dans le devenir de l'institution de la Compagnie de Jésus et dans celui de l'institution sociale en général, comme institution en devenir, inaccomplie. 


\section{$-1-$}

\section{L'impossible rappel des faits}

On pourrait écrire simplement ceci : le 15 août 1534, sept individus se retrouvent dans une chapelle située au flanc de la colline de Montmartre ${ }^{2}$ et s'y engagent à une action commune qui débouchera sur l'approbation pontificale d'un nouvel institut religieux, la Compagnie de Jésus, en 1540, dont ces sept hommes, augmentés de trois autres, seront les premiers membres. Pourquoi ne pas l'écrire ? Parce que tous les choix terminologiques de cet énoncé, pourtant réduit ici à une forme minimale, modèlent la signification du fait, négativement (comment qualifier ces «individus"? Six laïcs et un prêtre? Trois castillans, un navarrais, un basque, un portugais et un savoyard? Six jeunes gens de dixneuf à vingt-huit ans et un septième homme plus âgé, d'une quarantaine d'années ? Sept étudiants?), et positivement : comment définir, comment décrire ce qui s'est passé dans cette journée d'août? Le terme d'« engagement» mobilise immédiatement un temps futur sans lui donner aucune limite: or, comme on le verra, la limitation de cet engagement infléchirait inévitablement et brutalement l'interprétation du vœu de Montmartre, mais, inversement, la levée de toute limite ne rend pas justice de l'ensemble des interprétations possibles. Le terme d'«action » est lui aussi porteur, cette fois-ci tout à fait positivement, d'une signification forte, en particulier dans son rapport à celui qui suit dans le rappel du fait que je viens de tenter: une action «commune ». À l'action commune s'associera tout naturellement, par exemple, une contemplation singulière, l'une et l'autre s'opposant à la contemplation commune, définissant ainsi la spécificité d'un ordre de clercs réguliers par rapport aux institutions monastiques traditionnelles, et faisant du vœu de Montmartre la première manifestation d'une nouvelle ère des communautés religieuses chrétiennes. La date d'août 1534, sitôt prononcée, voisine aussitôt avec d'autre dates, que le rappel des faits ne pourra pas ne pas prendre sous son regard, l'affaire des placards, en particulier, et l'aggravation brutale des tensions politiques et religieuses à Paris et en France, une référence très certainement active dans la Compagnie de Jésus et dans l'investissement de ce mois d'août 1534². Enfin, et surtout, la mise en relation de l'événement du 15 août 1534 et de l'approbation de la Compagnie de Jésus place l'un et l'autre dans une série continue et permet de comprendre comment, jusqu'à aujourd'hui, le vœu de Montmartre a pu s'instaurer comme le moment de fondation du nouvel Ordre, dont l'approbation de 1540 marquerait l'aboutissement institutionnel ${ }^{4}$.

Or s'il nous semble difficile de produire un tel énoncé, c'est parce que la documentation historique du vœu de Montmartre se caractérise, peut-être avec une évidence trop aveuglante pour avoir été bien clairement perçue, par deux traits fondamentaux.

D'une part, cet événement n'est attesté par aucun document contemporain: non seulement il ne donne lui-même lieu à aucun texte - par exemple à un engagement écrit auquel les sept participants de la cérémonie auraient souscrit - et il n'est attesté par aucune chronique immédiate, ou proche. On n'en trouve la trace que beaucoup plus tard, lorsque une partie au moins des sept (l'un d'entre eux, le plus âgé, n'en fera jamais directement mention) convoque ce souvenir. Certes celui-ci, dans des termes sur lesquels nous reviendrons, apparaît comme une préfiguration de la fondation, six années plus tard, de la Compagnie de Jésus, mais cette préfiguration est rétrospective. Il va de soi que 
toute préfiguration relève, pour reprendre les concepts de Paul Ricoeur ${ }^{5}$, de la mise en intrigue refigurante d'une série d'événements donnés, mais il va moins de soi que ce processus ne tienne pas seulement de l'interprétation, mais de l'élaboration d'un épisode, dont aucun élément constituant ne peut être, en toute rigueur, tenu, tout au moins à un certain stade, en amont du travail de l'interprétation.

D'autre part, il s'agit d'une scène dont les acteurs sont définitivement les seuls témoins (sur le modèle d'une autre préfiguration chrétienne, l'Annonce faite à Marie), ou pour le dire autrement et doublement, dont les seuls témoins sont aussi les acteurs et dont les acteurs ne sont que les témoins, en devant alors irrésistiblement confier à ce mot la signification forte d'un dévoilement, d'un aveu, d'un martyr. Le vœu de Montmartre - mont des martyrs - est une cérémonie secrète, mais ce secret entre dans une cohérence, c'est-à-dire aussi une vraisemblance profonde avec l'acte-témoignage du martyr, dévoilement à soi de sa propre foi devant Dieu, dévoilement à soi en Dieu dans la grâce de la mort, et non pas seulement (la justification et l'économie théologique du martyr sont dans cette articulation) exemple de foi devant les hommes.

7 Cohérence et vraisemblance qui fondent ce qui pourrait, ce qui aurait pu être fable, au point de rencontre, nous semble-t-il d'une logique générale du témoignage comme passage à l'acte et d'une logique spécifique du phénomène de fondation: l'énoncé d'une fondation est nécessairement rétrospectif, il ne peut pas être contemporain d'un acte qui l'autorise, il est dans l'après-coup, car la fondation elle-même ne souffre d'antériorité que celle avec laquelle elle fait radicalement rupture; mais, du même coup, si cette rétrospection est la seule énonciation possible de l'événement de fondation, elle est aussi le lieu de son insistance, de sa revenance comme forme de présence.

8 Cette hypothèse théorique, qui suffirait à créditer le vœu de Montmartre d'une consistance historique, se trouve empiriquement secondée par une troisième caractéristique de ce vœu, résultante analytique des deux premières (et que je n'ai pour cette raison pas annoncée comme telle). On a donc affaire à une scène rétrospective, dont les seuls témoins sont les acteurs : ces acteurs sont plusieurs. C'est aussi parce qu'il n'y a pas concordance entre les différents récits conservés de l'événement que la préfiguration échappe à l'emprise de la rétrospection, qu'un champ de possibles se trouve réouvert. L'hétérogénéité des formes du vœu de Montmartre manifesterait l'insistance du moment de la fondation dans la mémoire de l'institution. Or l'ambivalence de cette proposition (selon que l'on porte son accent sur la fondation ou sur sa mémoire) se trouve projetée, par une double voie qui réfléchit et rejoue cette ambivalence, au cœur du problème du vœu religieux.

9 D'une part, si l'on met en regard ce qui s'est passé à Paris le 15 août 1534, en lui donnant la forme la plus générale ou la plus commune possible aux différents récits, soit un projet d'association, et la définition, générale elle aussi, du vœu de religion, soit la conjonction d'une appartenance communautaire et d'une tutelle autoritaire, on s'aperçoit que le vœu de Montmartre creuse l'écart entre les deux pôles de cette définition : c'est dans le nom de vœu rétrospectivement donné à ce qui s'est passé à ce moment-là que se manifeste l'exercice d'une tutelle autoritaire sur une appartenance communautaire. Les récits de l'événement, dont l'hétérogénéité - pas de point de vue un, pas de surplomb autorisé - est ici essentielle, construisent le fait en même temps qu'ils opèrent et qu'ils signalent le coup de force de sa dénomination comme vœu.

10 D'autre part, ce coup de force est d'autant plus remarquable que la référence du vœu de religion peut apparaître par ailleurs comme un modèle de préfiguration rétrospective. 
Fracturée d'une part, la référence du vœu de religion suture d'autre part le projet et sa résolution en combinant prolepse et analepse, anticipation et rétrospection. Emile Benveniste a montré dans des pages décisives ${ }^{6}$ que le vœu religieux en général - que je distingue ici du vœu de religion en particulier comme conjonction d'une appartenance communautaire et d'une tutelle autoritaire - pouvait représenter ce qu'il est demandé à la puissance divine d'exaucer, donc un vou d'obtenir, et ce qui est promis à cette puissance, vœu d'accomplir. Ces deux formes de vœu sont articulées : je formule un vœu à l'exaucement duquel je répondrai par l'accomplissement d'un autre vœu. Le vœu de religion proprement dit transforme doublement cet énoncé. Il identifie, quant à leur substance, les deux vœux, et conduit ainsi à une formulation proprement contradictoire : je m'acquitte d'un vœu promis parce que j'ai obtenu l'exaucement de ce même vœu; il lève cependant la contradiction en différenciant dans le temps ce qu'il identifie quant à la substance, en différant le vœu d'accomplir (qui revient à prononcer ses vœux) du vœu d'obtenir (qui revenait lui aussi, déjà, à faire ses vœux, ou plus précisément à faire le vœu de faire ses vœux). D'où l'importance, nous semble-t-il, de l'ensemble des dispositifs de «mise en intrigue» du processus de la prise de vœu dans les institutions religieuses particulières, et qui enracinent de fait une part essentielle de leur propre différenciation juridique positive dans ce travail ; d'où aussi l'attention que nous portons, entre d'autres aspects ${ }^{7}$, au vœu de Montmartre, qui fait revenir dans le temps, en tendant ses deux pôles comme la corde d'un arc, un seul et même vœu, le seul dont nous puissions dire en toute rigueur qu'il fut prononcé, celui que rappellent, dans le moment où ils le rappellent, les six plus un compagnons d'août 1534.

\section{Le silence du récit d'ignace}

11 Engagement et acquittement, liaison et déliaison, aliénation et libération: nous reviendrons pour finir sur les effets de cette spirale du vœu, à l'échelle de la Compagnie de Jésus en particulier et de l'institution religieuse en général par rapport à l'institution sociale. Mais il nous faut retenir maintenant, toujours du côté du fait historique du vœu de Montmartre et de ses apories, une troisième détermination de l'enquête, au-delà d'une recherche sur le nom de vœu donné à ce fait, et sur le temps de vœu attaché à sa préfiguration rétrospective, ou plus exactement au point de rencontre de ces deux orientations. Dans le Récit qu'il dicte, à la fin de sa vie (entre 1553 et 1555) à Luis Gonçalvez de Camara ${ }^{8}$, Ignace de Loyola fait silence sur le vœu de Montmartre. Ce silence porte à son comble ce que j'ai appelé plus haut le coup de force de la dénomination de cet événement comme vœu, en rejetant celui-ci dans l'innommable, mais donne parallèlement le moyen d'une interprétation de ce trou dans le récit, en en faisant le lieu d'une intensification extrême du régime temporel et narratif analeptique et proleptique ${ }^{9}$ qui caractérise par ailleurs le Récit d'Ignace dans son ensemble - d'une intensification jusqu'à la syncope de la préfiguration et de la rétrospection dans une conflagration de présence. Pour le dire globalement, le Récit, donné au passé, progresse sur un fil interrompu par deux séries d'attaques, les unes en direction d'un passé antérieur, et qui concernent pour l'essentiel la mémoire des "péchés passés », les autres en direction de l'horizon de futur de ce qui est raconté au passé, selon deux modalités successives d'un bout à l'autre du Récit, d'abord entre-coupé de prolepses projectives (ce que le jeune Ignace projette d'accomplir) puis de prolepses projectives (ce qu'il a accompli depuis). Or le glissement de l'une à l'autre de ces formes est par ailleurs soutenu par une autre dérive, massive, de la mémoire vers le projet en général. La première partie du Récit [1-11] 
est presque entièrement scindée et tendue entre mémoire et prolepse projective, la seconde [12-76] est presque entièrement au passé de récit, la troisième [77-97] réarticule les retours de mémoire sur des prolepses objectives - pour conduire dans le dernier moment du Récit à l'ouverture de celui-ci sur du présent, sur le temps présent de la Compagnie de Jésus instituée: le Récit s'interrompt en effet sur le seuil de cette institution, quand «d'autres pourront raconter » [98]. Car le temps présent est aussi un temps commun. Ce mouvement général, de l'analepse vers la ou les prolepses et l'ouverture, hors-cadre, à un présent commun, nous reconduit par trois voies au vœu de Montmartre.

D'abord parce que ce vœu, soustrait au passé de récit, mais aussi, comme on va le voir, à un retour de mémoire, s'inscrit en creux dans le corps du Récit comme un effet de présence, qui traverserait le texte, depuis l'étrange surgissement du présent dans les mots : « il se souvient spécialement de Dona Teresa Cardeñas » [60], qui n'est plus le plusque-passé de la mémoire, rappelé au passé, des "péchés passés ", jusqu'au suspens du récit devant le présent, c'est-à-dire très précisément dans une praesens, une imminence de présence - en passant par le non-passé, le passé qui ne passe pas du vœu de Montmartre, que les six compagnons d'Ignace rappelleront encore au soir de leur propre vie.

Six compagnons-deuxième voie-dont l'épisode manquant du vœu marquerait la première entrée dans le Récit d'Ignace (si l'on écarte ici pour la rapidité de l'analyse la "première équipe » qui entoure Ignace avant son séjour parisien, première équipe sur laquelle le Récit revient longuement et qui devrait également nous retenir plus : elle contribue fortement, en effet, à creuser l'écart de l'appartenance communautaire et de la tutelle autoritaire, et à placer le problème du vœu religieux au centre du Récit). Le temps commun prend donc d'abord la forme d'une trouée du récit unique.

Penchons-nous plus immédiatement enfin sur cette trouée :

Les médecins lui disaient seulement [à Ignace] qu'il ne lui restait rien d'autre que l'air natal qui puisse lui venir en aide. Les compagnons aussi lui conseillaient la même chose et insistèrent beaucoup auprès de lui.

Et déjà, à cette époque, tous avaient décidé de ce qu'ils devaient faire, à savoir : aller à Venise et à Jérusalem et dépenser leur vie pour être utile aux âmes, et si la permission ne leur était pas donnée d'aller à Jérusalem, retourner à Rome et se présenter au Vicaire du Christ pour qu'il les emploie là où il jugerait que ce serait davantage à la gloire de Dieu et plus utile pour les âmes. Ils s'étaient aussi proposés d'attendre pendant un an une embarcation à Venise; et s'il n'y avait pas d'embarcation pour le Levant pendant cette année, ils seraient relevés du vœu d'aller à Jérusalem (liberati del voto di Jerusalem) et se rendraient auprès du pape, etc ${ }^{10}$.

On note en passant, en écho direct à nos remarques générales, que ces lignes s'achèvent, non pas sur un engagement, mais sur une délivrance. Je reviendrai plus loin sur ce passage dans son ensemble, pour ne m'intéresser ici qu'à ce qui sépare "[...] ils insistèrent beaucoup auprès de lui » de "Et déjà, à cette époque [...]... ». Contrairement à ce que l'on pourrait penser, ce n'est pas du vœu de Montmartre que ces derniers mots annoncent le rappel: en effet, comme d'autres témoignages - en particulier celui de Pierre Favre - nous le diront, la décision « de ce qu'ils devaient faire » est déjà prise à l'heure de cette cérémonie, qui, déjà, renouvelle cette décision (avant que le vœu proprement dit ne soit renouvelé deux fois dans les deux années suivantes). On n'a donc pas affaire ici à un récit «analeptique » de l'épisode, mais on n'a pas affaire non plus à une annonce de cet épisode à l'aval de la décision prise. Le paragraphe suivant du Récit 
commence ainsi : «On était alors en 1535 [...] ». Le vœu de Montmartre se situe donc entre le moment, rappelé, dans lequel "tous avaient décidé», et celui qui suit immédiatement les lignes citées :

À la fin, le pèlerin se laissa persuader par les compagnons parce que, de plus, ceux qui étaient espagnols avaient certaines affaires à arranger qu'il pourrait régler. Et ils convinrent que, une fois qu'il irait bien, il irait arranger leurs affaires et se rendraient ensuite à Venise, où il attendrait les compagnons.

On était alors en 1535 [...].

Le récit du vœu de Montmartre est soumis à une double poussée, proleptique par le rappel de la décision prise, dans l'avenir de laquelle le vœu est encore, analeptique par la date indiquée, qui renvoie août 1534 dans un passé antérieur. J'appelle ici syncope ${ }^{11}$ ou effet de présence le produit de cette double poussée ou la résultante de ces deux forces, qui neutralise le temps du récit. En réalité, le paragraphe dans lequel la décision est rappelée peut être considérée comme tenant lieu du récit du vœu, comme le représentant de cet impossible récit au neutre (ni au passé, ni au futur), comme la représentation narrative d'une présence.

17 Le silence du Récit d'Ignace sur le vœu de Montmartre fait, nous semble-t-il, clairement apparaître les enjeux de la polyphonie du souvenir de ce vœu dans la première histoire de la Compagnie de Jésus. Première entrée des nouveaux compagnons, l'épisode manquant, écho interne, dans le corps du Récit, de son suspens au seuil de l'Ordre approuvé (en 1540), fait revenir le passé en présence dans une forme vide. C'est un temps commun qui ne se coule pas dans un récit unique, c'est un temps commun qui survit au récit unique de l'institution constituée - qui lui survit en 1555, date du Récit, et qui lui survit encore lorsque les compagnons du fondateur, souvent longtemps plus tard, en rappellent l'événement au souvenir de l'Ordre.

\section{$-\|$}

Repartons du Récit d'Ignace :

[...] tous avaient décidé de ce qu'ils devaient faire, à savoir : aller à Venise et à Jérusalem et dépenser leur vie pour être utile aux âmes, et si la permission ne leur était pas donnée d'aller à Jérusalem, retourner à Rome et se présenter au Vicaire du Christ pour qu'il les emploie là où il jugerait que ce serait davantage à la gloire de Dieu et plus utile pour les âmes. Ils s'étaient aussi proposés d'attendre pendant un an une embarcation à Venise; et s'il n'y avait pas d'embarcation pour le Levant pendant cette année, ils seraient relevés du vœu d'aller à Jérusalem (liberati del voto di Jerusalem) et se rendraient auprès du pape, etc.

18 Selon ces lignes, la cérémonie du 15 août 1534 (ici, je le rappelle, passée sous silence), a confirmé un projet (celui d'«aller à Venise et à Jérusalem»), le projet d'un sacrifice ("dépenser leur vie pour être utile aux âmes»), projet soutenu par la célébration actuelle, ce 15 août, comme nous l'apprenons par d'autres témoignages, d'une messe ; la cérémonie confirme également un premier serment, celui de se tenir au projet tracé pendant l'espace d'un an passé à Venise, et la promesse conditionnelle d'un second serment, celui de se rendre auprès du pape ; entre ces deux serments, se trouve introduit une sorte de contrat, celui de ne se trouver engagé par le projet de Jérusalem que sous la condition qu'avant un an il soit possible de passer au Levant. Comme nous l'avons déjà noté plus haut, un vœu n'est convoqué qu'au sujet de Jérusalem et pour en retenir le caractère temporaire. On a donc curieusement affaire à la combinaison d'une série de 
formes de lien, entre chacun des individus et leur fidélité à eux-mêmes et entre ces individus et leur fidélité réciproque, une combinaison qui les compose sans les réduire, c'est-à-dire sans en produire une synthèse sous la forme du vœu de religion - projet, sacrifice, promesse, serment et contrat, ramassés ensemble parce que subsumés sous un principe d'autorité12.

\section{Les troubles de la première historiographie}

19 La difficulté de ce passage du Récit d'Ignace et, après lui, de ceux des autres témoins de la cérémonie, les uns et les autres constituant le premier matériau des histoires de la Compagnie de Jésus produites à partir des années 1570, affleure dans deux circonstances précises de ces récits secondaires: la première concerne le lieu de la cérémonie, la seconde l'amplitude dans le temps, du « vœu » prononcé alors.

Dans les réponses qu'il fait, vers 1597-1600, aux questions posées par Nicolao Lancitio sur le sujet de la vie d'Ignace de Loyola, comme dans les critiques qu'il adresse, dans la même période, à la première biographie du fondateur par Pedro de Ribadeneira, Oliverius Manareus, alors provincial de Belgique, mais qui avait séjourné à la maison professe de Rome du vivant d'Ignace, distribue la cérémonie du vœu de Montmartre sur deux lieux : les compagnons auraient prononcé leurs vœux de pauvreté et de chasteté (composante absente du récit d'Ignace) dans la chapelle de Montmartre, puis se seraient engagés au voyage de Jérusalem et à l'obéissance du pape,

[...] vota ierosolymitanae peregrinationis et obedientiae praestandae Pontifici circa missiones,

dans l'église de Notre-Dame des Champs,

[...] ad templum beatae Virginis, quae a Campis nomen habet, in suburbiis S. Germani) $)^{13}$.

Manareus organise ainsi entre l'un et l'autre temples, la formulation, ou plus précisément la « rénovation » des deux vœux monastiques de la pauvreté et de la chasteté et celle du projet des sept compagnons, qu'il modifie sur deux points essentiels, puisque la nature contractuelle du vœu d'aller à Jérusalem disparaît en même temps que la nature hypothétique du vœu de "se rendre auprès du pape », selon les termes du Récit d'Ignace. Il est cependant remarquable que la construction du récit de Manareus ne permette pas de trancher entre deux possibilités : celle d'une montée de Notre-Dame des Champs à Montmartre, et par conséquent d'une postulation des vœux monastiques, ou inversement celle d'une descente de Montmartre vers Notre-Dame des Champs, et par conséquent d'une position préalable de ces mêmes vœux. Il présente en effet les deux lieux, en terminant par Montmartre. Puis,

[...] in eo templo vota castitatis et paupertatis renovabant, et in altero illo templo ediderant vota ierosolymitanae peregrinationis, etc.

La référence monastique est posée, mais son rang, si l'on peut dire, dans la cérémonie elle-même (et spécialement dans la cérémonie, puisqu'il s'agit par ailleurs d'une renovatio ), n'est pas arrêté, et n'est donc pas arrêté avec elle le registre du vœu d'obéissance au pape, dernier ou premier vœu, dernier vœu monastique (du côté de l'obéissance en général, ici absent) ou premier vœu jésuite ( $d u$ côté du pèlerinage et dans son inspiration).

21 Le récit de Pedro de Ribadeneira, censuré sur ce point par Oliverius Manareus ${ }^{14}$, se tient donc à un seul lieu pour le vœu de Montmartre. En revanche, il élargit le spectre temporel de la séquence, en deux points. 

espagnole (1583), on ne trouve pas l'énoncé

[...] c'est de là que prit son origine la rénovation des vœux dont on use dans la

Compagnie avant la profession

pas plus qu'on ne trouve dans cette version latine ceci, quelques lignes plus haut dans la version espagnole, après le rappel, parmi les «vœux » faits à Montmartre (et qui sont tous devenus des "vœux», ce qui n'était pas le cas, on s'en souvient, dans le Récit d'Ignace), celui de

[...] s'offrir au pape pour que celui-ci dispose d'eux librement :

[...] et c'est de là que prit son origine le quatrième vœu des missions, que nous

offrons au souverain pontife quand nous faisons profession dans la Compagnie.

Or la version espagnole introduit aussi, sur un registre tout différent au premier abord, un autre complément, essentiel, nous semble-t-il, par rapport à l'interprétation du vœu de Montmartre et de son destin troublé dans la première historiographie de la Compagnie de Jésus,

Ils firent vœu de s'employer au profit spirituel du prochain et d'aller en pèlerinage à Jérusalem, avec cette condition que, arrivés à Venise (llegados a Venecia), ils attendraient pendant une année entière une embarcation [...].

Par cette seule incise, qui réintroduit d'ailleurs une donnée présente dans le Récit ignatien, le texte espagnol marque une discontinuité entre le temps du vœu de Montmartre et celui de Venise, lui-même directement et sans plus de rupture articulé à celui de Rome et à l'approbation de l'Ordre. Le compte à rebours de cette approbation ne commence pas à Paris, comme le texte latin pouvait le laisser calculer, mais à Venise. Il ne s'agit plus ici de l'amplitude temporelle intensive du vœu de Montmartre (vœu de se vouer, vœu rénové), mais d'une amplitude extensive : jusqu'où, par le moyen de ce vœu de Montmartre, peut-on ou ne peut-on pas faire remonter la chronologie de la fondation ? À cette question, le texte espagnol de la Vita apporte une double réponse, très précisément travaillé entre l'une et l'autre versions, en faisant d'une part, par diverses voies, du vœu 
de Montmartre le modèle ou l'archétype des formes institutionnelles du vœu de religion dans la Compagnie de Jésus instituée, mais en soustrayant d'autre part l'événement de ce vœu à une chronologie continue qui, en provoquant une régression, dans le temps, du processus de fondation, ne peut que réduire et fragiliser d'autant la durée de vie de l'institution. Plus la gestation aura été longue, plus l'institution apparaîtra brève ${ }^{17}$.

De ce système d'écluse ${ }^{18}$, par lequel l'institution ramène à elle le temps de la fondation en même temps que ce temps de fondation se trouve, du même coup, remis en présence, la discontinuité de la Vita de 1583 tente le contrôle. Mais ce contrôle est d'autant plus précaire qu'il en va comme nous l'avons vu de la structure même du vœu de Montmartre en particulier et du vœu de religion en général, comme préfiguration rétrospective, liaison et déliaison, de ne trouver sa règle que dans un parcours ininterrompu de l'arc du temps, qui seul, de prononciation en confirmation, de rénovation en rénovation, assure la liaison, diffère l'acquittement, mais fait aussi revenir, par dessous ce nom de vœu, ce qui s'est passé le 15 août 1534.

\section{Le vœu de montmartre selon ses témoins}

Venons enfin, très brièvement au regard de ce que mériterait l'ensemble de ces textes discrets et complexes ${ }^{19}$, aux récits des autres acteurs/témoins de la cérémonie du 15 août $1534^{20}$. Quatre documents ont été conservés, représentant cinq compagnons sur six (la lettre de Diego Laynez associant Alfonso Salmerón) et pouvant être répartis en deux catégories: Pierre Favre (1506-1542) et Laynez-Salmerón (1512-1565, 1515-1585) donnent leur témoignage en 1542 et 1547; Simão Rodriguez (1510-1577) et Nicolás Bobadilla (1509-1590) en 1577 et 1590. Les deux premiers sont proches de l'événement, et antérieurs même au Récit d'Ignace; les deux derniers sont beaucoup plus tardifs. Le témoignage de Simão Rodriguez est suscité par le général Everard Mercurian, qui succède dans cette fonction à Diego Laynez (1558-1565) et à François de Borgia (1565-1572); celui de Nicolás Bobadilla intervient, comme un testament, dans les derniers mois de sa vie. On proposera ici quelques éléments autour d'un paradoxe central : les témoignages tardifs remettent en jeu dans le vœu de Montmartre un polymorphisme que les témoignages les plus précoces tendaient au contraire à réduire. La fonction de préfiguration de cet événement dans la mise en intrigue de l'histoire de l'Ordre conquiert sa consistance et son autonomie en proportion de l'ampleur de la rétrospection dont elle est le fruit. Il n'y a pas, de ce point de vue, érosion mais au contraire capitalisation de la force fondatrice ou refondatrice de la cérémonie du 15 août 1534.

Pierre Favre écrit dans les premières pages de son Mémorial :

Le jour de Sainte Marie d'août, en cette même année [1534], déjà unis par une même détermination (in eadem eramus determinatione) et formés aux Exercices (sauf Maître François [Xavier], qui ne les avait pas encore faits, bien qu'il partageât notre projet (determinatione)), nous allâmes à Notre Dame de Montmartre, près de Paris, $\mathrm{y}$ faire chacun le vœu (votum) de partir pour Jérusalem à la date qui nous serait indiquée, et de nous placer au retour sous l'autorité du Pontife Romain, et aussi de commencer chacun, au temps marqué (die assignato), à quitter nos parents et nos filets, gardant seulement un peu d'argent pour la route [...] Nous retournâmes tous au même endroit, les deux années suivantes, en ce même jour de Notre-Dame d'août, afin de nous confirmer dans ces résolutions (eam determinationem) pour lesquelles nous nous trouvions chaque fois en progrès selon l'esprit ${ }^{21}$. 
contenu du vœu est ici donné dans sa double entrée, selon une conjonction reprise beaucoup plus tard par Ribadeneira dans sa Vita Ignatii : on n'ira pas à Rome si l'on ne va pas à Jérusalem, mais au retour de Jérusalem. Or cette conjonction est ambivalente : certes, elle pose le terme romain comme nécessaire, et non pas comme possible, et fait de la fondation de l'Ordre le relais et la transformation du geste pèlerin. Dans un temps proche encore de l'approbation officielle de la Compagnie de Jésus, l'interprétation du texte de Favre peut et doit aller dans cette direction. Il n'empêche que, s'il pose ainsi le terme romain, Favre ne maintient pas moins, ou, plus encore, ne définit pas moins le pèlerinage, rétrospectivement et bien que celui-ci n'ait pas eu lieu, comme un préalable nécessaire à l'entrée dans Rome. Certes, le retour de Terre Sainte est supposé, mais l'aller s'impose. Il faut maintenir en veille cette autre lecture, car elle fait ressortir du texte une trame plus souterraine, celle qui conduit de «détermination» en "détermination »- le mot est rappelé trois fois dans ce court texte - et prend en écharpe le terme-une seule fois requis - de "vœu », et donne du coup un relief particulier à l'introduction progressive du vœu de pauvreté dans le «vœu de Montmartre»: le vœu de pauvreté n'est pas un substrat de la " détermination », il ne viendra, ou plus précisément ne commencera de venir qu'«au temps marqué ». L'« arme secrète » du commencement, d'un temps zéro dont le temps du vœu parisien est tout à la fois le terme exclu et la clé, arme secrète dont nous avons vu toute la tradition postérieure jouer - dans ce que j'ai appelé le système d'écluse du vœu de Montmartre-, est donc ici mobilisée sur le sujet capital de l'articulation entre l'engagement des vœux monastiques et la «détermination » des futurs membres fondateurs de la Compagnie de Jésus.

La lettre-récit écrite par Diego Laynez (avec la collaboration probable d'Alfonso Salmerón) en juin 1547 (l'année de l'entrée en fonctions au secrétariat d'Ignace de Loyola de Juan de Polanco, futur premier historiographe de l'Ordre) a déjà retenu notre attention. Sur le versant de la critique (autrement dit des conditions de possibilité) de l'institution, la question du commencement et de la régression du temps zéro y est posée, sur le versant-prioritaire dans les textes de cette première période-de l'insertion institutionnelle des formes primitives de l'association, le motif de la rénovation des vœux y est souligné.

Reprenons maintenant les deux paragraphes consacrés par la lettre, directement ou indirectement, au vœu de Montmartre :

Nous sommes restés à Paris pour achever les études, en nous fixant comme délai le jour de la fête de la Conversion de saint Paul de 1537, bien que par la suite, en raison des choses qui survinrent à cause des guerres et de je ne sais quelles autres choses, nous soyions partis le 15 novembre 1536. À Paris, nous nous sommes affermis [...] par le vœu que nous avions fait de nous consacrer au service du Seigneur, dans la pauvreté, à partir du délai dont il a été parlé ; ce vœu, nous l'avons chacun renouvelé et confirmé le jour de Notre Dame d'août à Sainte-Marie de Montmartre, où nous l'avions fait une première fois après nous être confessés et avoir communié.

[...] Nous sommes ainsi arrivés à Venise le 8 janvier 1537.

[...] Et parce que notre intention à Paris n'était pas de créer une congrégation, mais de nous consacrer dans la pauvreté au service de Dieu Notre Seigneur et au bien du prochain en prêchant et en servant dans les hôpitaux, etc., nous fîmes le vœu, quelques années avant de partir pour réaliser notre intention, d'aller si nous le pouvions aux pieds du pape, vicaire du Christ, et de lui demander la permission d'aller à Jérusalem, et si la possibilité se présentait, de rester là-bas pour y progresser, si Notre Seigneur, en était servi, et de faire progresser les autres, fidèles et infidèles ; et s'il n'y avait pas la possibilité d'aller là-bas à Jérusalem dans l'année 
ou, en y allant, de rester là-bas, nous avions précisé dans le vœu que nous n'avions pas l'intention de nous obliger davantage à y aller, mais de retourner auprès du pape et de nous mettre sous son obéissance, en allant là où il nous enverrait ${ }^{22}$. d'une part, le rite de rénovation est premier, il renvoie le premier vœu dans un plus-queparfait, non sans pousser le vœu renouvelé vers le présent (par la forme gérondive) :

[...] nous avions fait [le vœu] de nous consacrer au Seigneur (con haber hecho el voto); ce vœu, nous l'avons renouvelé et confirmé (renovando y confirmando), [là] où nous l' avions fait une première fois (donde primero lo hicimos) ${ }^{23}$.

À l'échelle d'un temps intensif, le vœu de Montmartre est déjà rétrospectif, il est déjà la préfiguration de sa rénovation; d'autre part, le récit développe ensuite sur une plus grande échelle le geste rétrospectif, à la manière, mais seulement à la manière d'une rénovation : c'est à Venise que se trouve rappelé le vœu de Montmartre - Venise, ville des possibles, ville des multiples déterminations. La rénovation (narrative) du vœu, à Venise, n'est pas - pas encore ou jamais tout à fait - un rite d'institution. La lettre de Laynez (et de Salmerón) se tient en position de bascule sur le pont de l'écluse dont le vœu de Montmartre règle les ouvertures.

Simão Rodriguez produit le plus long témoignage conservé sur le vœu de Montmartre, dans le deuxième chapitre de son Commentarium de origine et progressu societatis Iesu (1577). Le chapitre s'ouvre sur une première « détermination »:

Ces sept Pères (certains avaient et d'autres n'avaient pas la même détermination ( porque ainda entam os otros nao tinhao a mesma determinaça $\left.{ }^{24}\right)$ ) persévérant [...], ils commencèrent (se começou) à se demander à quel moment ils quitteraient Paris pour commencer à réaliser leurs désirs (per començar a dar principio a sus deseos). Et parce que, conformément à leur détermination d'employer leur vie (Dieu les faisant revenir de Jérusalem) au service et l'aide du prochain pour son salut, il leur sembla que ce ministère demandait plus de lettres qu'ils n'en avaient encore [...]

$\mathrm{Au}$-delà de cette détermination (determinaçao), il leur parut convenable, pour donner plus de fermeté aux choses, de tous faire vœu (voto) ${ }^{25}$ de pauvreté, de chasteté et d'aller à Jérusalem, puis, Dieu les faisant revenir de là-bas, d'employer leur vie au service de Dieu [... ${ }^{26}$.

Suit l'exposé de l'ensemble des «déterminations » des premiers Pères : comme on vient de le voir, Simão Rodriguez y incorpore d'emblée les vœux de chasteté et de pauvreté (bien que ce dernier "ne s'entende pas pendant leur séjour d'études à Paris", mais suppose cependant d'emblée le refus de «toute aumône pour les messes qu'ils diraient »- rétrospection aventurée sans doute en 1534 : seul Favre est alors prêtre); il propose par ailleurs un long développement, dont il est semble-t-il le seul à se souvenir, sur la décision au scrutin majoritaire (a major parte delles), de l'installation à Jérusalem ou du retour ; la décision, ce retour effectué ou le voyage étant demeuré impossible (« un an après l'arrivée à Venise »), de se rendre à Rome ne relève pas explicitement d'un même mode de scrutin : « tous iraient alors au pape », et dans le latin :

[...] nullo obstricti voto Romam (quod faciendum etiam erat hominibus iam reversis Hierosolyma) contenderent ${ }^{27}$.

Cette nouvelle phase reste cependant largement ouverte: ils demanderont au pape licence de pouvoir partout " prêcher ", " confesser ", " donner la communion", et de pouvoir «prêcher parmi les infidèles ». Il est remarquable que le projet circonscrit de Jérusalem donne lieu à une décision majoritaire, alors que l'offrande faite au pape de leur "service", si elle doit s'imposer aux compagnons, ne recouvre aucun engagement déterminé (le pape ne devra "approuver»-le terme pèse son poids ${ }^{28}$-que leur " détermination ») : on n'y retrouve pas même le présupposé d'une cohésion du groupe. 


\section{pape :}

Ce qui a été déclaré ci-dessus fut la première trace (traça / lineamento) que la sagesse divine donna de notre Compagnie, et l'on fit ce vœu, la première fois qu'on le fit, l'année (si je me souviens bien) 1534, le 15 août, jour de Notre-Dame d'août [...] $]^{29}$.

Le vœu de Montmartre vient toucher, voire mordre - » on » le fera d'autres fois - le trait de fondation de la Compagnie, revenant par surprise, presque par effraction - » ce qui a été déclaré » devient «ce vœu»-chahuter la chronologie : la première détermination était antérieure, bien antérieure même (le chapitre s'ouvre sur sa confirmation) à 1534, la dernière préfigure rétrospectivement la Compagnie dans la «trace » du contenu du vœu de 1534. Or, à l'incertitude soudaine, et surprenante après tant de détail, de Rodriguez sur cette date (se bem me sembra), me semble répondre, à l'extrémité du chapitre, un retour de mémoire inverse, mais un retour qui continue de mordre le trait, de bousculer le temps, un retour qui est une venue en présence :

Je certifie à $\mathrm{V}[\mathrm{otre}] \mathrm{P}[\text { aternité }]^{30}$ que ces Pères ont fait ce sacrifice de soi à Dieu en se livrant à lui avec tant de vérité et un tel renoncement de leurs volontés propres et avec une telle dévotion et une telle confiance dans leur miséricorde, que plusieurs fois depuis, en y repensant, une nouvelle dévotion et une nouvelle admiration ont grandi en moi. Que grâce soit rendue à Dieu pour toutes ses œuvres, et parce que c'est notre mémoire qu'il nous fasse miséricorde (et quia memor fuit nostri faciens nobiscum misericordiam suam $)^{31}$.

Entre ces deux pôles, Rodriguez raconte, avec plus de précision qu'aucun autre témoin, la cérémonie du vœu de Montmartre. Ce récit nous semble contenir la clé du passage de la mémoire incertaine au souvenir continué, parce que s'y donne secrètement à entendre ce qu'aucun témoin de l'événement n'a rappelé (c'est l'évidence que l'enquête peine à voir, qui fait ciller les yeux) : la formule du vœu de Montmartre. Et que, ce faisant, la tradition jésuite renoue, mais en marge de l'institution, dans ses fondations, avec une tradition coextensive à l'histoire du monachisme chrétien, la syncope de l'énoncé du vœu, toujours soustrait à la lettre des règles, toujours tenu dans le lieu silencieux des paroles dites, parce que l'acte de cet énoncé fonde et refonde chaque fois l'institution, parce qu'il est son secret de fabrication :

On choisit (elegeram) et on détermina (determinarao) que le lieu de ce vou (este voto) serait une colline appelée Mont des Martyrs [...] Et pour pouvoir faire ce sacrifice avec plus de dévotion, on s'y prépara par un jeûne [...]. À ce voeu, quand il se fit les deux années suivantes le même jour et dans le même lieu [...], le P. Ignace ne fut pas présent, mais tout se fit selon ses avis et conseils [...]. Quand ils furent tous réunis dans cet ermitage et sans personne de dehors, le Père Favre dit la messe et, avant de leur donner la communion, tenant devant eux le très Saint Sacrement, chacun à part soi, le genou à terre et le cœur en Dieu, fit son vœu d'une voix distincte et que tous entendirent, et tous reçurent ensuite le très Saint Sacrement; et le P. Favre, se tournant vers l'autel, fit lui aussi, avant de communier, le même vœu d'une voix distincte que tous entendirent ${ }^{32}$.

La Crono-historia de la Compania de Jesus en la Provincia de Toledo (1710), de Bartolomeo de Alcazar, qui est en réalité, dans sa première partie, une histoire générale de l'Ordre, trouve à ce problème une solution originale. L'auteur fait d'abord le récit de la rencontre des six compagnons et d'Ignace qui précède la cérémonie du vœu, et lors de laquelle le contenu de ce vœu aurait été décidé. Il place à ce moment-là dans la bouche d'Ignace un long discours, rapporté au style direct, dans lequel ce contenu est énoncé. Puis,

[...] le saint se tut [...] et tous se rallièrent à son dictamen, 
sans s'être eux-mêmes directement exprimés. Lorsque, quelques jours plus tard, les compagnons se réunissent une nouvelle fois à Montmartre,

[...] à commencer par Saint Ignace, tous firent leurs vœux dans sa formule

déterminée, selon la forme dont ils avaient convenu ${ }^{33}$.

Ainsi l'énoncé du vœu est-il produit sans l'être : il est répété (ou préfiguré) par le maitre, mais son passage dans la bouche des disciples reste tenu dans l'acte de son énonciation ${ }^{34}$.

Dernier témoignage de notre série, celui du vieux Nicolas Bobadilla dans le récit de sa vie, l'année de sa mort, en 1590. Mémoire chancelante, sans doute, voire même perte de mémoire, vie au présent, en mal, en marge ou en rupture d'histoire. Bobadilla fait du vœu d'aller à Jérusalem l'article unique de l'engagement de Montmartre :

Il [Bobadilla] fit le vœu de se rendre en pauvreté au saint sépulcre de Jérusalem ( voverat se iturum in paupertate ad sanctum sepulchrum hierosolimitanum), fréquentant la confession et la communion avec d'autres maîtres dévots; nombre d'entre eux devinrent compagnons et frères de la Compagnie de Jésus et tous avaient fait le vœu de Jérusalem (eran voti hierosolimitani) et vouèrent publiquement ce vœu ( publice voverunt votum) au Mont des Martyrs le jour de la fête de l'Assomption de Marie $^{35}$.

Cette formulation radicale n'est, nous semble-t-il, nullement infirmée ${ }^{36}$, mais tout au contraire explicitée dans une lettre écrite par Bobadilla quelques mois plus tôt, le 11 août 1589, au général des jésuites, Claudio Aquaviva :

Me souvenant, comme je m'en souviens souvent, de cette sainte fête de l'assomption de la glorieuse Madone, et surtout dans ma vieillesse, je ne peux m'empêcher d'écrire à V[otre] P[atemité], en considérant comment, ce jour-là, les premiers Pères de la Compagnie nous fîmes (fescemo) le vœu d'aller à Jérusalem, au Mont des Martyrs près de Paris, lequel vœu la divine providence, quae est abyssus multa ["qui est un grand abîme», Psaume 34, 7], a changé en d'autres vœux meilleurs et plus fructueux de pèlerinages en religion ${ }^{37}$.

\section{- III -}

Nicolás Bobadilla écrivait déjà le 31 août 1569 , vingt ans avant son autobiographie testamentaire, au général François de Borgia :

Ce n'est pas sans mystère que V[otre] $\mathrm{P}$ [aternité] m'a écrit si longuement de Frascada le jour de l'Assomption de Notre Dame la Vierge Marie, pour laquelle j'ai une grande dévotion, car c'est un tel jour que commença notre Compagnie au Mont des Martyrs, près de Paris, où nous fîmes les premiers vœux pour aller à Jérusalem tous les dix. Cette fête est le cep et l'origine de la Compagnie : « Regardez Abraham, votre Père et Sara qui vous a mis au monde ", disait Dieu par son prophète [Esaie 51, 2], en leur rappelant le Père et la Mère dont ils descendaient. Et c'est ainsi que nous devons faire, nous autres de la Compagnie, et surtout ceux d'entre nous qui étions là (los que alli nos allamos), quand le Christ et sa mère nous prirent pour leurs fils, et pour les pères d'une si grande Compagnie ${ }^{38}$.

On suspendra la recherche en cours, à partir de ce dernier texte, sur deux perspectives pour le développement de l'interprétation du vœu de Montmartre. La lettre de Bobadilla à François de Borgia pose en effet le problème dans des termes très clairs.

D'une part, elle confirme, parce qu'elle manifeste la réitération indéfinie - jusqu'à la mort-du vœu d'août 1534, les limites de la traduction institutionnelle du renouvellement de ce vœu, comme renouvellement de l'institution de l'Ordre, réinstauration perpétuelle du procès de sa fondation, dans la procédure normée (qui, comme on l'a vu, tira pourtant sa légitimité de cette référence) de la renovatio voti. On 
peut écrire, au titre d'hypothèse, que le vœu de Montmartre, en tant que préfiguration rétrospective de l'Ordre, se donne dans la première tradition de la Compagnie de Jésus instituée comme la modalité narrative, irréductiblement excessive, de la formulation juridique du vœu de religion, pour ce qui concerne la rénovation des vœux comme pour ce qui concerne la procédure du vœu simple public, vœu du vœu, en particulier si l'on considère, dans cette procédure, la virtualité de l'autorité, le retardement de la décision d'autorité par rapport à l'énonciation du vœu d'être autorisé.

D'autre part, comme le montre bien, avec profondeur, la lettre de Bobadilla, le vœu de Montmartre refait affleurer ce que l'institution scelle, mais aussi recèle, par-dessous l'appartenance autorisée à l'Ordre : qu'en est-il du lien des pères et des fils? Comment les fils deviennent-ils des pères, cette "Votre Paternité » à laquelle s'adresse Bobadilla, en 1569 comme en 1589 ? Comment les pères, inversement, redeviennent-il ou refusent-ils de redevenir des fils dans une généalogie reconstituée ? Comment l'ordre religieux ne rétablit-il jamais tout à fait le formidable ébranlement auquel l'événement de la fondation soumet la loi familiale, en faisant des pères et des fils, dans le moment de cet ébranlement, des individus absolus, aussi neutres en regard de cette généalogie qu'était silencieusement neutre, dans le Récit d'Ignace, l'événement du vœu de Montmartre soumis à la double poussée du passé et de l'avenir? Il s'agit là de bien autre chose, de quelque chose d'une toute autre portée que les réglages - et les règlements de compte-internes d'une institution religieuse déterminée; il s'agit du rapport de l'institution religieuse en général, et de la décomposition-recomposition du lien social qui fait l'essence de son invention, avec l'institution sociale en général. Le lien du vœu, dans son indétermination, ouvert, dans son acte - dans le secret de sa formulation, tel que le récit de Simão Rodriguez en rejoue l'enjeu - au projet, au sacrifice, à la promesse, au contrat, au serment, produit le vœu de l'institution religieuse comme vœu d'institution ou vœu d'association réglée en général et, du même coup, renvoie toute institution sociale effective à la particularité de ses procédures. Le lien du vœu, tel que le vœu de Montmartre en produit et en reproduit l'indétermination, l'ouverture, le polymorphisme, suspend et refonde, refonde et retient encore en suspens l'institution en général, dans ses particularités, comme garantie du lien social. Cela, tous les religieux dont nous avons fait comparaître ici les témoignages le savent, et ils en usent, ad intra et ad extra.

\section{NOTES}

1. Le « vœu de Montmartre " a été relativement peu étudié dans la période récente. Signalons surtout trois articles de Pedro Leturia, «Jerusalen y Roma en los designios de San Ignacio de Loyola » (1929), «Importancia del ano 1538 en el cumplimiento del "Voto de Montmartre" » et «El voto de San Ignacio en Montmartre », tous deux datés de 1940, recueillis dans Pedro Leturia, Estudios ignacianos 1957, I, p. 181-200, p. 201-223, et II, p ; 405-411 ; Manuel Ruiz Jurado, « Ce qui se passa à Montmartre le 15 août 1534 », Centrum Ignatianum Spiritualis, 1985, p. 15-34.

2. Le lieu du vœu de Montmartre fut probablement la dite "chapelle des martyrs ", située en contrebas de l'abbaye des Bénédictines blanches qui occupait le sommet de la butte: André Ravier, Ignace de Loyola à Paris, Paris, Vie chrétienne, 1999 ; l'auteur s'appuie sur le plan de ville 
contemporain d'Olivier Truschet et Germain Hoyau (1530-1550). Comme on le verra, les témoignages restent cependant imprécis sur le séjour d'Ignace et de ses compagnons à l'intérieur de la chapelle : le vœu a-t-il été prononcé dans le bâtiment en surface ou dans la crypte ? André Ravier, qui opte pour la seconde hypothèse, s'éloigne sur ce point des sources conservées. Or l'indétermination relative du lieu nous semble importante : le vœu de Montmartre oscille par elle entre l'édifice et ses fondations.

3. On rappelera, aussi pour mémoire, l'article célèbre de Lucien Febvre, «Une date, 1534 : la Messe et les placards ", 1945, in Au cœur du XVI siècle religieux, Paris, 1957.

4. On en prendra pour exemple la chronologie établie par Jean Lacouture à la fin du premier volume de Jésuites, Paris, Seuil, 1991, dans laquelle l'auteur note, pour l'année 1534: «Vœu de Montmartre des sept fondateurs », avant d'indiquer, pour 1540 : « Paul III approuve la Compagnie de Jésus », p. 494.

5. Paul Ricoeur, Temps et récit, t. I, Paris, Seuil, 1983, en part. p. 55-129. Le problème qui nous occupe ici peut apparaître comme un cas très particulier (mais qui est peut-être aussi une structure spécifique des phénomènes de fondations religieuses) que l'on pourrait renvoyer à cet énoncé général : «Dès qu'une histoire est bien connue - et c'est le cas de la plupart des récits traditionnels ou populaires, aussi bien que celui des chroniques nationales rapportant les événements fondateurs d'une communauté - suivre l'histoire c'est moins enfermer les surprises ou les découvertes dans la reconnaissance du sens attaché à l'histoire prise comme un tout qu'appréhender les épisodes eux-mêmes bien connus comme conduisant à cette fin ", (p. 105).

6. Emile Benveniste, Le Vocabulaire des institutions indo-européennes, t. II, Paris, Minuit, 1969, p. 233-243.

7. En particulier, toujours dans la Compagnie de Jésus, la formulation intermédiaire entre le vœu simple et le vœu solennel du vœu simple public (Pierre-Antoine Fabre, "Prononcer ses vœux ", L'inactuel, 1995, 4, p. 221-230), ou encore les cérémonies de rénovation des vœux (enquête en cours, sur la base d'un grand nombre d'écrits, demeurés manuscrits pour l'essentiel, consacrés à la théorie de cette pratique dans la seconde moitié $\mathrm{du} \mathrm{XVI}{ }^{\mathrm{e}}$ siècle).

8. Ignace de Loyola, « Récit », in Écrits, éd. Maurice Giuliani, Paris, Desclée de Brower, 1991.

9. Je recours ici comme plus haut aux outils narratologiques forgés naguère par Gérard Genette, et qui conservent une robuste efficacité. On trouvera une analyse beaucoup plus complète du Récit d'Ignace, qui n'a jamais été étudié comme un texte, dans Pierre-Antoine Fabre, De la fondation. Recherches sur la première Compagnie de Jésus, chap. v (à paraître).

10. Ignace de Loyola, op. cit., p. 1062 [85]. Le texte original de cette partie du Récit, probablement dictée par Luis Gonçalvez de Camara à partir des propos entendus d'Ignace, à un scripteur génois au cours de son voyage vers Lisbonne dans les derniers mois de l'année 1555, est en langue italienne.

11. L. Marin, De la représentation, Paris, « Hautes Etudes », Paris, Gallimard/Seuil, 1994, p. 364-376. 12. On demande ici au lecteur d'admettre au titre d'hypothèse de travail cette proposition, dont le développement excèderait évidemment de loin le cadre de cet article, mais qui est pour nous au cœur de la réflexion engagée en compagnie d'Alain Boureau sur le sujet du vœu religieux.

13. Monumenta Historica Societatis Iesu (MHSI), Fontes Narrativi, III, Rome, 1960, p. 438. Olivierus Manareus établit la continuité entre les deux lieux par saint Denis, capturé à Notre-Dame des Champs et martyrisé à Montmartre. Je remercie Jean-Marie Le Gall de m'avoir signalé, à l'occasion de ses recherches sur l'histoire de saint Denis, l'importance croissante de la référence dyonisienne dans la tradition jésuite française du vœu de Montmartre, qui intervient plus chez Manareus comme l'instrument d'une articulation des deux lieux que dans le cadre d'un développement propre à conférer, comme cela sera le cas par la suite (par exemple dans la Vie de saint Denis du jésuite Etienne Binet en 1629), une légitimité française à la Compagnie de Jésus.

14. La version produite dans les censures de la Vita Ignatii Loyolae de Pedro de Ribadeneira est presque identique (MHSI, FN IV, Rome, 1965, p. 990). Elle mobilise cependant en sa faveur, assez 
curieusement, le témoignage de Pierre Favre, dont on verra plus loin qu'elle ne «bi-localise » nullement le vœu de Montmartre. Erreur de lecture, liée au fait que Favre nomme la chapelle du Mont des Martyrs Notre-Dame de Montmartre ? Il est tout aussi possible que le Mémorial de Favre ait donné lieu, pour cette raison ou pour une autre, à des transcriptions ou à des récits intermédiaires dont Manareus tirerait son information. Cette deuxième hypothèse alourdirait le dossier de ce premier trouble dans la tradition jésuite de la seconde moitié du Xvi ${ }^{\mathrm{e}}$ siècle.

15. MHSI, FN IV, op.cit., p. 232-233. On trouve aussi cette forme dans un texte presque contemporain de la version latine de la Vita, le Chronicon de Juan de Polanco, ancien secrétaire d'Ignace de Loyola, amorcé autour de 1573 : [...] proximorum saluti procurandi, et ad condictum tempus Hierosolymam profiscendi (MHSI, Chron. Pol. I, Madrid, 1894, p. 50).

16. Ibid.

17. Ribadeneira prend le contre-pied, en revanche, de la lettre écrite en juin 1547 par Diego Laynez et Alfonso Salmeron (à laquelle nous reviendrons), qui ne fait nulle mention de Venise, mais seulement, indirectement, de Rome, où ils devront obtenir du pape la permission du passage en Terre Sainte. Dans ce scénario, Rome intervient en amont de Venise, dans l'horizon du pèlerinage, et non pas en aval, dans l'horizon de la fondation de l'Ordre. Pedro Leturia (op. cit., p. 207-208) remarque cette discordance, mais n'en tire pas toutes les conséquences pour ce qui concerne la fonction du vœu de Montmartre dans les choix historiographiques des premiers récits de fondation. Leturia poursuit, en réalité, un autre objectif, qui n'est cependant pas sans lien avec notre problème : il s'attache en effet à retarder l'année d'attente jusqu'au printemps 1537 (c'est-à-dire jusqu'au second séjour des compagnons à Venise après leur passage par Rome, précédé d'un premier passage à Venise), et donc à la placer entre le printemps 1537 et le printemps 1538. Dans cet autre scénario, note Leturia, "c'est l'année 1538 et non pas l'année 1537 qui en termine avec les obligations de Montmartre (cierra las obligaciones de Montmartre) ", p. 221. Le compte à rebours de la fondation semble remonter jusqu'au temps de Paris; Laynez et Salmerón écrivent: «nous fîmes le vœu [...] que s'il n'y avait pas dans l'espace d'un an la possibilité d'aller de là-bas vers Jérusalem (de ir allá a Jerusalem dentro de un año) » mais, en réalité, ce reflux se répercute en aval sur une prolongation des «obligations de Montmartre », qui ne relèvent pas du projet d'institution.

18. Le moment vénitien dans son ensemble est essentiel pour toute cette analyse, puisque s'y joue, à travers le rapport du projet de pèlerinage et du projet missionnaire, le temps d'avant et le temps d'après, le passage de l'avant à l'après par la transformation du pèlerinage en mission. Mais le vœu de Montmartre est étroitement lié à cette question plus générale, en particulier, justement, parce que le vœu de pèlerinage reste irréductible au vœu de religion et contribue à faire du moment vénitien un moment de bascule et un carrefour d'interprétations. Je me permets de renvoyer sur ce point à Pierre-Antoine Fabre, "Ils iront en pèlerinage". L'expérience du pèlerinage selon l'Examen général des Constitutions de la Compagnie de Jésus et selon les pratiques contemporaines ", in Rendre ses voeux. Identités pèlerines dans l'Europe moderne, Philippe Boutry, Pierre-Antoine Fabre et Dominique Julia (éd.), Paris, ÉHESS, 2000.

19. J'écarte provisoirement d'un bloc toute une partie de l'enquête, naturellement à conduire, sur la trajectoire des « témoins » du vœu au sein de la Compagnie de Jésus et la contextualisation institutionnelle de leur récit, pour me limiter à une coupe transversale des formes du vœu dans leurs différences et leurs écarts.

20. Notons d'emblée que les trois futurs membres de la Compagnie de Jésus, le picard Paschase Broet, le provençal Jean Codure et le savoyard Claude Jay, qui auraient participé à la « rénovation » du vœu de Montmartre de 1536 selon le Mémorial de Pierre Favre, éd. Michel de Certeau, Paris, Desclée de Brower, 1959, p.117, n'ont laissé aucun témoignage direct de cette dernière cérémonie. Doit-on en conclure, dans le prolongement de notre analyse de la Vita espagnole de Ribadeneira, que l'importance de la rénovation du vœu de Montmartre relèverait effectivement d'une construction rétrospective de cette rénovation comme préfiguration de la « 
renovatio voti » dans la Compagnie de Jésus instituée ? La mort rapide de Jean Codure (en 1541) et de Claude Jay (en 1552) enlève sans doute un argument à cette hypothèse. Le cas de Paschase Broët (mort en 1562), ainsi que le témoignage précoce de Pierre Favre (mort en 1546), tendraient au contraire à renforcer celle-ci.

21. Pierre Favre, op. cit., p. 117, trad. de Michel de Certeau ; le texte latin original est dans MHSI, Fabrii Monumenta, Rome, 1914, p. 496.

22. MHSI, FN I, op. cit., p. 102-103, p. 110-112.

23. La version latine, contemporaine, de la même lettre force le trait en introduisant un deuxième renvoi au premier vœu "émis": denique voto quo obstrinximus nos omnibus rebus renunciandi et mancipandi nos in perpetuum Domini nostri obsequium et condictam diem. Et quando hoc votum emisimus, etiam inter nos sanximus quotannis semel renovandum atque confirmandum esse a nobis die Assumptionis B. Virginis Mariae consacrato in aede B. Mariae, quae Mons Martyris vulgo dicitur, ubi primum votum hoc emisimus.

24. Simão Rodriguez vient de citer les trois futurs membres de la cérémonie de renouvellement des vœux, absents le 15 août 1534. L'ambiguïté de la parenthèse, qui semble porter un jugement négatif sur cette deuxième vague, est levée dans la version latine contemporaine : nondum enim reliqui se dederant in eorum societatem.

25. Le texte latin choisit curieusement pour determinaçao : disputatio, qui rompt la continuité du passage au voto (votum).

26. MHSI, FN III, op. cit., p. 21.

27. Id., p. 22-23.

28. Le texte latin évite d'ailleurs de le reprendre : [...] ut intelligeret Summus Pontifex paratos esse se [...], Id., p. 22-24.

29. Id., p. 24-25.

30. Simão Rodriguez adresse son mémoire au général de l'Ordre (Everard Mercurian en 1577). Nous reviendrons plus loin et pour finir sur cette adresse au général des premiers Pères.

31. Id., p. 26-27. Rodriguez cite Tobie 8, 18, auquel il ajoute et quia memor fuit nostri.

32. Id., p. 25-27.

33. Bartolomeo de Alcazar, Crono-historia de la Compania de Jesus en la Provincia de Toledo, Madrid, 1710 , t. I, p. 35.

34. L'exemplaire consulté de la Crono-historia de Bartolomeo de Alcazar (à la bibliothèque Sèvres, Paris) s'ouvre sur deux feuillets manuscrits collés au folio, dans lesquels le scripteur-qui pourrait bien être l'auteur du livre: on lit sur la couverture du volume ces deux lignes manuscrites las dos ojas m.s. que estan al principio in testimonium malignitas mea-se livre à une critique sévère de la Compagnie de Jésus dans l'état dans lequel elle est aujourd'hui, et des erreurs contenues dans le livre, qui apparaissent moins comme des erreurs de l'auteur, que comme des déformations transmises par la tradition et révélatrices de la décadence de la Compagnie. Or ces deux feuillets accordent une place centrale au vœu de Montmartre, et consacrent près de deux pages sur quatre - bien longtemps avant Pedro Leturia - à la discussion de la chronologie des événements, et au temps d'attente, beaucoup trop bref, des compagnons à Venise, pour conclure ceci (alors qu'il défend par ailleurs la mémoire des "premiers religieux", trahie par cette présentation des faits) : La guerre [empêchant les navires de prendre la mer pour Jérusalem] peut bien excuser ceux qui manquent d'espérance : mais il faut bien peu de ferveur pour restreindre ses désirs au port ou aux ports de Venise, et de renoncer si vite [au départ pour la Terre Sainte] en s'écartant du premier motif qui avait motivé la Fondation (f. 2 r).

35. MHSI, Bobadillae Monumentae, Rome, 1970, p. 615. C'est, selon la suite du récit de Bobadilla, le pape Paul III qui, «après l'année 1538 ", convainc Ignace et ses compagnons, en leur déclarant : L'Italie est une bonne et vraie Jérusalem, [de renoncer] à ce qu'ils avaient jusqu'alors toujours gardé dans leur cour et dans leur prière [...] accomplir leur vœu de pèlerinage à Jérusalem, p. 616.

36. M. Ruiz Jurado plaide pour la « nuance » qu'apporterait cette lettre, art. cit., p. 43. 
37. MHSI, Bob. Mon., op. cit., p. 602.

38. Id., p. 498. 